\title{
Double Sumudu Transform Iterative Method for One- Dimensional Nonlinear Coupled Sine-Gordon Equation
}

\author{
Alemayehu Tamirie Deresse iD \\ Department of Mathematics, Faculty of Natural Science, Mizan Tepi University, Tepi, Ethiopia \\ Correspondence should be addressed to Alemayehu Tamirie Deresse; alemayehutamire006@gmail.com
}

Received 12 October 2021; Accepted 10 December 2021; Published 5 January 2022

Academic Editor: Antonio Scarfone

Copyright (C) 2022 Alemayehu Tamirie Deresse. This is an open access article distributed under the Creative Commons Attribution License, which permits unrestricted use, distribution, and reproduction in any medium, provided the original work is properly cited.

\begin{abstract}
In this paper, the combined double Sumudu transform with iterative method is successfully implemented to obtain the approximate analytical solution of the one-dimensional coupled nonlinear sine-Gordon equation (NLSGE) subject to the appropriate initial and boundary conditions which cannot be solved by applying double Sumudu transform only. The solution of the nonlinear part of this equation was solved by a successive iterative method, the proposed technique has the advantage of producing an exact solution, and it is easily applied to the given problems analytically. Two test problems from mathematical physics were taken to show the liability, accuracy, convergence, and efficiency of the proposed method. Furthermore, the results indicate that the introduced method is promising for solving other types of systems of NLPDEs.
\end{abstract}

\section{Introduction}

The system of coupled sine-Gordon equations in the form

$$
\left\{\begin{array}{l}
u_{t t}-u_{x x}=-\delta^{2} \sin (u-v), \\
v_{t t}-\alpha^{2} v_{x x}=\sin (u-v),
\end{array} \quad \alpha>0, \delta>0,\right.
$$

was introduced by Khusnutdinova and Pelinovsky [1]. The coupled sine-Gordon equations generalize the FrenkelKontorova dislocation model [2,3]. System (1) with $\alpha=1$ was also proposed to describe the open states in the DNA double helix model [4]. This type of equation has been receiving an enormous amount of attention due to the presence of soliton solutions, and it has applications in the propagation of fluxons in Josephson junctions between two superconductors [5], the motion of a rigid pendulum attached to a stretched wire [6], etc.

Recently, many researchers used various methods to get the solution of system (1). Li et al. [7] presented a lattice Boltzmann model for the two-component system of coupled sine-Gordon equations by using the coupled mesoscopic
Boltzmann equations and the Chapman-Enskog multiscale expansion, while the macroscopical governing evolution system can be recovered correctly by selecting suitable discrete equilibrium distribution functions and the amending functions. In paper [8], the authors used a rational exponential ansatz to derive the exact solutions of a coupled sineGordon equation. The simplest equation method has been used for finding the exact solutions of coupled sineGordon equations by Zhao [9]. In papers [10-12], Zhao et al. obtained some new solutions of coupled sine-Gordon equations including Jacobi elliptic function solutions, hyperbolic function solutions, and trigonometric function solutions by the Jacobi elliptic function expansion method, the hyperbolic auxiliary function method, and the symbolic computation method. Sadighi et al. [13] employed the homotopy perturbation method (HPM) for solving both sine-Gordon and coupled sine-Gordon equations. In paper [14], the authors obtained numerical solution of inhomogeneous systems of sine-Gordon equation (1) by finite difference method with fixed-point iteration.

In 2018, the authors of [15] studied the coupled sineGordon equations in nonlinear optics, which describe the 
propagation of an optical pulse in fiber waveguide, and they derived the new exact solutions of the problem through the use of the well-organized modified Kudryashov method. For more related research about solving the sine-Gordon equations, interested readers may refer to $[16-18]$.

Since many application problems in science and engineering are formulated by nonlinear partial differential equations, new techniques are required to solve those problems efficiently. The Sumudu transform is an integral transform similar to the Laplace transform, introduced in the early 1990 s by Watugala [19] to solve differential equations and control engineering problems. Recently, this method is used to solve differential equations like nonlinear boundary value problems [20] and the Cauchy problem for the wave equation in one dimensional space [21]. In [22], the Sumudu transform is applied to arbitrary powers Dumont bimodular Jacobi elliptic functions for arbitrary powers. Furthermore, Watugala [23] has extended the Sumudu transform to two variables with emphasis on solutions to partial differential equations. Ahmeda et al. [24] studied the convergence properties of double Sumudu transformation and used it to find the exact solution of the Volterra integropartial differential equation. In the paper [25], the authors' applied the double Sumudu transform linked with the Adomian decomposition method or with variational iteration method to find the analytical solution of nonlinear fractional partial differential equations. Kiwne and Sonawane [26] present the proofs of some theorems of double Sumudu transform with application in solving partial differential equations like one-dimensional heat equation, Poisson equation, and second-order hyperbolic equations of two variables. The advantage of the double Sumudu transform is it gives a rapid convergence of the exact solution without any restrictive assumption of the solution compared to other known methods (see [27]). Unfortunately, this transform fails to solve nonlinear partial differential equations like other integral transforms; to solve this problem, this transform is often combined with other methods like the variational method [28], reduced differential transform method [29], double integral transform (Laplace-Sumudu transform) method [30], Adomian decomposition method $[25,31]$, and homotopy perturbation method [20, 32].

The main aim of this work is to apply the double Sumudu transform method coupled with the new iterative method (NIT) proposed by Daftardar-Gejji and Jafari in [33] to find an exact/approximate solution of the nonlinear coupled sine-Gordon equation. The new iterative method (NIM) has been extensively used by many researchers for the treatment of linear and nonlinear ordinary and partial differential equations of integer and fractional order (see [33-37]). The method converges to the exact solution if it exists through successive approximations. The nonlinear term in the equation is expanded in terms of DaftardarGejji and Jafari polynomials (see [38, 39]).

In the present study, we consider the nonlinear initial boundary value problem (IBVP) for the one-dimensional coupled sine-Gordon equation [14] in the following form:

$$
\left\{\begin{array}{l}
u_{t t}-u_{x x}=-\sin (u-v)+f(x, t), \\
v_{t t}-v_{x x}=\sin (u-v)+h(x, t),
\end{array}\right.
$$

subject to the initial conditions

$$
\begin{cases}u(x, 0)=\varphi_{1}(x), & u_{t}(x, 0)=\varphi_{2}(x) \\ v(x, 0)=\varphi_{3}(x), & v_{t}(x, 0)=\varphi_{4}(x)\end{cases}
$$

and boundary conditions (Cauchy-type BCs)

$$
\left\{\begin{array}{l}
u(0, t)=g_{1}(t), u_{x}(0, t)=g_{2}(t) \\
u(0, t)=g_{3}(t), u_{x}(0, t)=g_{4}(t)
\end{array}\right.
$$

within the problem domain of $\Omega=\{x: a \leq x \leq b\}$ and $t$ $>0$.

The remaining parts of this paper are structured as follows. In Section 2, we begin with some basic definitions, properties, and theorems of the double Sumudu transform method. Section 3 illustrates the details of the new iterative method and its convergence. Section 4 presents how to obtain an approximate analytical solution of the coupled SGE using the DSTIM. In Section 5, we apply the proposed method to two illustrative examples in order to show its liability, convergence, and efficiency. Finally, concluding remarks are given in Section 6.

\section{A Brief Introduction of Double Sumudu Transform (DST)}

Definition 1 (see $[24,40]$ ). Sumudu transform of a function $f(t)$ is defined for all real numbers $t>0$ as the function $S($ $p)$, given by

$$
S_{t}[f(t)]=F(p)=\frac{1}{p} \int_{0}^{\infty} e^{-(t / p)} f(t) d t=\lim _{j \longrightarrow \infty} \frac{1}{p} \int_{0}^{j} e^{-(t / p)} f(t) d t
$$

Definition 2 (see [30]). The inverse Sumudu transform of a function $F(p)$ is denoted by the symbol $S^{-1}[F(p)]=f(t)$ and is defined by the Bromwich contour integral

$$
S_{t}^{-1}[F(p)]=f(t)=\lim _{T \longrightarrow \infty} \frac{1}{2 \pi i} \int_{\gamma-i T}^{\gamma+i T} e^{t / p} F(p) d p .
$$

Definition 3 (see $[24,40,41])$. Let $f(x, t)$ be a function that can be expressed as a convergent infinite series and let $(x, t$ ) $\in \mathbb{R}_{+}{ }^{n}$, then the double Sumudu transform of the function $f(x, t)$ in the positive quadrant of the $x t$-plane is given by

$$
S_{x} S_{t}[f(x, t)]=F(k, p)=\frac{1}{k p} \int_{0}^{\infty} \int_{0}^{\infty} e^{-(x / k+t / p)} f(x, t) d p d k,
$$

where $x, t \geq 0$, and $k, p$ are the transform variables for $x$ and $t$, respectively, whenever the improper integral is convergent. 
Definition 4 (see $[42,43]$ ). The inverse double Sumudu transform $S_{x}^{-1} S_{t}^{-1}[F(k, p)]=f(x, t)$ is defined by the following form:

$S_{x}^{-1} S_{t}^{-1}[F(k, k)]=f(x, t)=\frac{1}{2 \pi i} \int_{\alpha-i \infty}^{\alpha+i \infty} \frac{1}{k} e^{x / k}\left[\frac{1}{2 \pi i} \int_{\gamma-i \infty}^{\gamma+i \infty} \frac{1}{p} e^{t / p} F(k, p) d p\right] d k$

Remark 5 (see $[26,40]$ ). If $f(x, t)$ is a continuous function having a second partial derivative, then double Sumudu transforms of partial derivative of the first and second are defined as follows:

Double Sumudu transform for the first partial derivative with respect to $t$ is given by

$$
\begin{aligned}
S_{x} S_{\mathrm{t}}\left[\frac{\partial f(x, t)}{\partial t} ; k, p\right] & =\frac{1}{k p} \lim _{j \longrightarrow \infty}\left[\int_{0}^{j} \int_{0}^{j} e^{-(x / k+t / p)} f(x, t) d t d x\right] \\
& =\frac{1}{p}[F(k, p)-F(k, 0)] .
\end{aligned}
$$

Double Sumudu transform for the first partial derivative with respect to $x$ is given by

$$
\begin{aligned}
S_{x} S_{t}\left[\frac{\partial f(x, t)}{\partial x} ; k, p\right] & =\frac{1}{k p} \lim _{j \longrightarrow \infty}\left[\int_{0}^{j} \int_{0}^{j} e^{-(x / k+t / p)} f(x, t) d \mathrm{t} d x\right] \\
& =\frac{1}{k}[F(k, p)-F(0, p)] .
\end{aligned}
$$

Double Sumudu transform for the second partial derivative with respect to $t$ is given by

$$
\begin{aligned}
S_{x} S_{t}\left[\frac{\partial^{2} f(x, t)}{\partial t^{2}} ; k, p\right] & =\int_{0}^{\infty} \int_{0}^{\infty} \frac{\partial^{2} f(x, t)}{\partial t^{2}} e^{-\left(x / k_{k}^{x}+t / p\right)} f(x, t) d t d x \\
& =\frac{1}{p^{2}} F(k, p)-\frac{1}{p^{2}} F(0, p)-\frac{1}{p} \frac{\partial F(0, p)}{\partial x} .
\end{aligned}
$$

Double Sumudu transform for the second partial derivative with respect to $x$ is given by

$$
\begin{aligned}
S_{x} S_{t}\left[\frac{\partial^{2} f(x, t)}{\partial x^{2}} ; k, p\right] & =\int_{0}^{\infty} \int_{0}^{\infty} \frac{\partial^{2} f(x, t)}{\partial x^{2}} e^{-(x / k+t / p)} f(x, t) d t d x \\
& =\frac{1}{k^{2}} F(k, p)-\frac{1}{k^{2}} F(k, 0)-\frac{1}{k} \frac{\partial F(k, 0)}{\partial t} .
\end{aligned}
$$

\subsection{Convergence Theorem of Double Sumudu Transform}

Lemma 6 (see [24]). Let $f(x, t)$ be a function of two variables continuous in the positive quadrant of the $x t$-plane. If the integral

$$
\frac{1}{k p} \int_{0}^{\infty} \int_{0}^{\infty} e^{-(x / k+t / p)} f(x, t) d t d x
$$

converges at $k=k_{0}, p=p_{0}$, then the integral converges for $k$ $<k_{0}, p<p_{0}$.

2.2. Existence and Uniqueness of the Double Sumudu Transform. If $f(x, t)$ is an exponential order, then $c$ and $d$ as $x \longrightarrow \infty, t \longrightarrow \infty$, and if there exists a positive constant $K$ such that for all $x>X, t>T$, then

$$
|f(x, t)|=K e^{c x+d t} .
$$

And we write $f(x, t)=O e^{c x+d t}$ as $\longrightarrow \infty, t \longrightarrow \infty$. Or, equivalently,

$$
\begin{aligned}
& \lim _{x \longrightarrow \infty, t \longrightarrow \infty} e^{-(x / k)-t / p}|f(x, t)|=K \lim _{x \longrightarrow \infty, t \longrightarrow \infty} e^{-(x / k-c) x-(t / p-d) t} \\
& =0, \quad \frac{1}{k}>c, \frac{1}{p}>d .
\end{aligned}
$$

And we write this as $f(x, t)=\mathrm{O}\left(e^{a x+b y+c t}\right)$ as $x \longrightarrow \infty$ and $t \longrightarrow \infty$.

The function $f(x, t)$ is called an exponential order as $x$ $\longrightarrow \infty, t \longrightarrow \infty$, and clearly, it does not grow faster than $K e^{c x+d t} x \longrightarrow \infty, t \longrightarrow \infty$.

Theorem 7 (existence). If a function $f(x, t)$ is continuous in every finite intervals $(0, X)$ and $(0, T)$ and of exponential order $e^{c x+d t}$, then the double Sumudu transform of $f(x, t)$ exists for all $1 / k$ and $1 / p$ provided that $\operatorname{Re}(1 / k)>c$ and $\operatorname{Re}($ $1 / p)>d$.

For the proof, see $[42,43]$.

Theorem 8 (uniqueness). Let $f(x, t)$ and $g(x, t)$ be continuous functions defined for $x, t \geq 0$ and having double Sumudu transforms $F(k, p)$ and $G(k, p)$, respectively. If $F(k, p)=G(k$ $, p)$, then $f(x, t)=g(x, t)$.

\subsection{Some Properties of Double Sumudu Transform}

Property 9 (linearity of TLT, $[42,43])$. If $f(x, t)$ and $g(x, t)$ are two functions of $x$ and $t$ such that $S_{x} S_{t}\{f(x, t)\}=F(k$, $p)$ and $S_{x} S_{t}\{g(x, t)\}=G(k, p)$, then

$$
\begin{aligned}
S_{x} S_{t} & \{\alpha f(x, t)+\beta g(x, t)\}=\frac{1}{k p} \int_{0}^{\infty} \int_{0}^{\infty} e^{-(x / k+t / p)}[\alpha f(x, t)+\beta g(x, t)] d t d x \\
& =\frac{1}{k p} \int_{0}^{\infty} \int_{0}^{\infty} e^{-(x / k+t / p)} \alpha f(x, t) d t d x+\frac{1}{k p} \int_{0}^{\infty} \int_{0}^{\infty} e^{-(x / k+t / p)} \beta g(x, t) d t d x \\
& =\frac{\alpha}{k p} \int_{0}^{\infty} \int_{0}^{\infty} e^{-(x / k+t / p)} f(x, t) d t d x+\frac{\beta}{k p} \int_{0}^{\infty} \int_{0}^{\infty} e^{-(x / k+t / p)} g(x, t) d t d x \\
& =\alpha S_{x} S_{t} f(x, t)+\beta S_{x} S_{t} g(x, t)=\alpha F(k, p)+\beta G(k, p),
\end{aligned}
$$

where $\alpha$ and $\beta$ are constants. 
Property 10 (change of scale property, [44]). If $f(x, t)$ and $g(x, t)$ are two functions of $x$ and $t$ such that $S_{x} S_{t}\{f(x, t)\}$ $=F(k, p)$ and $S_{x} S_{t}\{g(x, t)\}=G(k, p)$, then

(i) $S_{x} S_{t}\{f(c x, d t)\}=(1 / c d) F(c k, d p)$,

(ii) $S_{x} S_{t}\{f(c x) g(d t)\}=(1 / c d) F(c k) G(d p)$,

where $c$ and $d$ are nonzero constants.

Property 11 (first shifting property). If $S_{x} S_{t}\{f(x, t)\}=F(u$, $v)$, then

$$
S_{x} S_{t}\left\{e^{c x+d t} f(x, t)\right\}=\frac{1}{(1-c k)(1-d p)} F\left(\frac{k}{1-c k}, \frac{p}{1-d p}\right) .
$$

Proof. from (7), we have

$$
\begin{aligned}
S_{x} S_{t}\left[e^{c x+d t} f(x, t)\right] & =\frac{1}{k p} \int_{0}^{\infty} \int_{0}^{\infty} e^{-(x / k+t / p)} e^{c x+d t} f(x, t) d t d x, \\
& =\frac{1}{k} \int_{0}^{\infty} e^{-(1 / k-c) x}\left[\int_{0}^{\infty} \frac{1}{p} e^{-(1 / p-d) t} f(x, t) d t\right] d x, \\
& =\frac{1}{k} \int_{0}^{\infty} e^{-(1 / k-c) x}\left[\int_{0}^{\infty} \frac{1}{p} e^{-(1 / p-d) t} f(x, t) d t\right] d x
\end{aligned}
$$

By using the integration by substitution method for the integral inside the bracket and the integral outside the bracket, we get

$$
S_{x} S_{t}\left\{e^{c x+d t} f(x, t)\right\}=\frac{1}{(1-c k)(1-d p)} F\left(\frac{k}{1-c k}, \frac{p}{1-d p}\right) .
$$

Similarly,

$$
L_{x} L_{y} L_{t}\left\{e^{-c x-d t}\right\} f(x, t)=\frac{1}{(1+c k)(1+d p)} F\left(\frac{k}{1-c k}, \frac{p}{1-d p}\right) .
$$

Property 12 (second shifting property, $[42,43])$. If $S_{x} S_{t}\{f(x$ $, t)\}=F(k, p)$, then

$$
S_{x} S_{t}\{f(x-c, t-d) H(x-c, t-d)\}=e^{-(c / k)-d / p} F(k, p),
$$

where $H(x, t)$ is the Heaviside unit step function defined by

$$
H(x-c, t-d)=\left\{\begin{array}{ll}
1, & x>c, t>d \\
0, & \text { otherwise }
\end{array}\right\}
$$

\section{The New Iterative Method}

Daftardar-Gejji and Jafari [33] have considered the following functional equation:

$$
u=N(u)+f
$$

where $N$ is a nonlinear operator in a Banach space such that $N: B \longrightarrow B$ and $f$ is a given element of the Banach space $B$. Here, $u$ is assumed to be the solution of equation (23) whose series expansion is given by

$$
u=\sum_{i=0}^{\infty} u_{i}
$$

The nonlinear operation $N$ can then be decomposed as

$$
N\left(\sum_{i=0}^{\infty} u_{i}\right)=N\left(u_{0}\right)+\sum_{i=1}^{\infty}\left\{N\left(\sum_{r=0}^{i} u_{r}\right)-N\left(\sum_{r=0}^{i-1} u_{r}\right)\right\} .
$$
lent to

Using Equations (24) and (25), Equation (23) is equiva-

$$
\sum_{i=0}^{\infty} u_{i}=f+N\left(u_{0}\right)+\sum_{i=1}^{\infty}\left\{N\left(\sum_{r=0}^{i} u_{r}\right)-N\left(\sum_{r=0}^{i-1} u_{r}\right)\right\} .
$$

From Equation (26), we define the following recurrence relation:

$$
\left\{\begin{array}{l}
u_{0}=f, \quad u_{1}=N\left(u_{0}\right), \\
u_{2}=N\left(u_{0}+u_{1}\right)-N\left(u_{0}\right), \\
u_{(n+1)}=N\left(u_{0}+\cdots+u_{n}\right)-N\left(u_{0}+\cdots+u_{(n-1)}\right), \quad n=1,2, \cdots .
\end{array}\right.
$$

Thus,

$$
\left(u_{1}+\cdots+u_{(n+1)}\right)=N\left(u_{0}+\cdots+u_{(n)}\right), \quad n=1,2, \cdots,
$$

and hence,

$$
\sum_{i=0}^{\infty} u_{i}=f+N\left(\sum_{i=0}^{\infty} u_{i}\right)
$$

Therefore, the $n^{\text {th }}$ term approximate solution of equation (24) is given by

$$
u=u_{0}+u_{1}+u_{2}+u_{3}+\cdots+u_{n-1}, \quad n>1 .
$$

\subsection{Convergence of the New Iterative Method}

Theorem 13. If $N$ is a continuously differentiable functional in a neighborhood of $u_{0}$ and $\left\|N^{(n)}\left(u_{0}\right)\right\| \leq M \leq e^{-1}$ for all $n$, then the series $\sum_{n=o}^{\infty} u_{n+1}$ is absolutely convergent [33]. 


\section{Double Sumudu Transform Coupled with Iterative Method}

To solve the system of sine-Gordon equation (2), first, we decompose the source function $f(x, t)$ into $f_{1}(x, t)$ and $f_{2}(x$, $t)$. The part $f_{1}(x, t)$ with the terms in equation (2) always leads to the simple algebraic expression while applying the inverse double Sumudu transform. The portion $f_{2}(x, t)$ is combined with the nonlinear term of equation (2) to avoid noise terms in the iteration process. Similarly, $h(x, t)=h_{1}(x, t)+h_{2}(x, t)$.

Applying the double Sumudu transform to both sides of the system of equation (2) and by using Table 1 and Definition 4 , we get

$$
\left\{\begin{array}{l}
\frac{1}{k^{2}} \bar{u}(k, p)-\frac{1}{k^{2}} \bar{u}(k, 0)-\frac{1}{k} \bar{u}_{t}(k, 0)-\frac{1}{p^{2}} \bar{u}(k, p)+\frac{1}{p^{2}} \bar{u}(0, p)+\frac{1}{p} \bar{u}_{x}(0, p) \\
=\bar{f}_{1}(k, s)-S_{x} S_{t}\left(\sin (u-v)-f_{2}(x, t)\right), \\
\frac{1}{k^{2}} \bar{v}(k, p)-\frac{1}{k^{2}} \bar{v}(k, 0)-\frac{1}{k} \bar{v}_{t}(k, 0)-\frac{1}{p^{2}} \bar{v}(k, p)+\frac{1}{p^{2}} \bar{v}(0, p)+\frac{1}{p} \bar{v}_{x}(0, p) \\
=\bar{h}_{1}(k, p)+S_{x} S_{t}\left(\sin (u-v)+h_{2}(x, t)\right),
\end{array}\right.
$$

where $\bar{u}, \bar{u}_{t}, \bar{u}_{x}, \bar{v}, \bar{v}_{t}, \bar{v}_{x} \bar{f}_{1}$, and $\bar{h}_{1}$ are Sumudu transforms of functions of two variables.

Multiplying both sides of each equation in system (31) by $k^{2} p^{2}$ yields

$$
\left\{\begin{array}{l}
p^{2} \bar{u}(k, p)-p^{2} \bar{u}(k, 0)-k p^{2} \bar{u}_{t}(k, 0)-k^{2} \bar{u}(k, p)+k^{2} \bar{u}(0, p)+p k^{2} \bar{u}_{x}(0, p) \\
=k^{2} p^{2} \bar{f}_{1}(k, s)-k^{2} p^{2} S_{x} S_{t}\left(\sin (u-v)-f_{2}(x, t)\right) \\
p^{2} \bar{v}(k, p)-p^{2} \bar{v}(k, 0)-k p^{2} \bar{v}_{t}(k, 0)-k^{2} \bar{v}(k, p)+k^{2} \bar{v}(0, p)+p k^{2} \bar{v}_{x}(0, p) \\
=k^{2} p^{2} \overline{h_{1}}(k, p)+k^{2} p^{2} S_{x} S_{t}\left(\sin (u-v)+h_{2}(x, t)\right)
\end{array}\right.
$$

Next, applying a single Sumudu transform to the systems of equations (3) and (4), we get

$$
\left\{\begin{array}{l}
\bar{u}(k, 0)=\overline{\varphi_{1}}(k), \bar{u}_{t}(k, 0)=\overline{\varphi_{2}}(k), \\
\bar{v}(k, 0)=\overline{\varphi_{3}}(k), \bar{v}_{t}(k, 0)=\overline{\varphi_{4}}(k),
\end{array}\right.
$$

$$
\left\{\begin{array}{l}
\bar{u}(0, p)=\overline{g_{1}}(p), \bar{u}_{t}(0, s)=\overline{g_{2}}(p), \\
\bar{v}(0, p)=\overline{g_{3}}(p), \bar{v}_{t}(0, s)=\overline{g_{4}}(p)
\end{array}\right.
$$

Here, $\overline{\varphi_{i}}$ and $\bar{g}_{i}(i=1,2,3,4)$ are Sumudu transforms of functions of single variables.

By substituting the systems of equations (34) and (33) into the systems of equation (32) and simplifying, we obtain

$$
\left\{\begin{array}{c}
\bar{u}(k, p)=\frac{k^{2} p^{2}}{k^{2}-p^{2}}\left[\begin{array}{c}
p^{2} \bar{u}(k, 0)+k p^{2} \bar{u}_{t}(k, 0)-k^{2} \bar{u}(0, p)-p k^{2} \bar{u}_{x}(0, p)-k^{2} p^{2} \bar{f}_{1}(k, p) \\
=-k^{2} p^{2} S_{x} S_{t}\left\{\left(\sin (u-v)-f_{2}(x, t)\right)\right\}
\end{array}\right] \\
\bar{v}(k, p)=\frac{k^{2} p^{2}}{k^{2}-p^{2}}\left[\begin{array}{c}
p^{2} \bar{v}(k, 0)+k p^{2} \bar{v}_{t}(k, 0)-k^{2} \bar{u}(0, p)-p k^{2} \bar{v}_{x}(0, p)-k^{2} p^{2} \overline{h_{1}}(k, p) \\
=k^{2} p^{2} S_{x} S_{t}\left\{\sin (u-v)+h_{2}(x, t)\right\}
\end{array}\right] .
\end{array}\right.
$$

Applying the inverse double Sumudu transform to the system of equation (35), we obtain

$$
\left\{\begin{array}{c}
u(x, t)=S_{x}^{-1} S_{y}{ }^{-1}\left\{\frac{k^{2} p^{2}}{k^{2}-p^{2}}\left[\begin{array}{c}
p^{2} \bar{u}(k, 0)+k p^{2} \bar{u}_{t}(k, 0)-k^{2} \bar{u}(0, p)-p k^{2} \bar{u}_{x}(0, p)-k^{2} p^{2} \bar{f}_{1}(k, p) \\
=-k^{2} p^{2} S_{x} S_{t}\left\{\left(\sin (u-v)-f_{2}(x, t)\right)\right\}
\end{array}\right]\right\}, \\
v(x, t)=S_{x}^{-1} S_{y}^{-1}\left\{\frac{k^{2} p^{2}}{k^{2}-p^{2}}\left[\begin{array}{c}
p^{2} \bar{v}(k, 0)+k p^{2} \bar{v}_{t}(k, 0)-k^{2} \bar{u}(0, p)-p k^{2} \bar{v}_{x}(0, p)-k^{2} p^{2} \bar{h}_{1}(k, p) \\
=k^{2} p^{2} S_{x} S_{t}\left\{\sin (u-v)+h_{2}(x, t)\right\}
\end{array}\right]\right\} .
\end{array}\right.
$$

Now, we start an iterative process. We assume that

$$
\left\{\begin{array}{l}
u(x, t)=\sum_{i=0}^{\infty} u_{i}(x, t) \\
v(x, t)=\sum_{i=0}^{\infty} v_{i}(x, t)
\end{array}\right.
$$

is the solution of the coupled nonlinear sine-Gordon equation (2).

Substituting the systems of equation (37) into the systems of equation (36), we obtain

$$
\left\{\begin{array}{l}
\sum_{i=0}^{\infty} u_{i}(x, t)=S_{x}^{-1} S_{y}^{-1}\left\{\frac { k ^ { 2 } p ^ { 2 } } { k ^ { 2 } - p ^ { 2 } } \left[\begin{array}{c}
\left.p^{2} \bar{u}(k, 0)+k p^{2} \bar{u}_{t}(k, 0)-k^{2} \bar{u}(0, p)-p k^{2} \bar{u}_{x}(0, p)-k^{2} p^{2} \bar{f}_{1}(k, p)\right] \\
\left.=k^{2} p^{2} S_{x} S_{t}\left\{f_{2}(x, t)-\sin \left\{\sum_{i=0}^{\infty}\left[u_{i}(x, t)-v_{i}(x, t)\right]\right\}\right\}\right],
\end{array}\right.\right. \\
\sum_{i=0}^{\infty} v_{i}(x, t)=S_{x}{ }^{-1} S_{y}{ }^{-1}\left\{\frac{k^{2} p^{2}}{k^{2}-p^{2}}\left[\begin{array}{c}
\left.p^{2} \bar{v}(k, 0)+k p^{2} \bar{v}_{t}(k, 0)-k^{2} \bar{u}(0, p)-p k^{2} \bar{v}_{x}(0, p)-k^{2} p^{2} \bar{h}_{1}(k, p)\right] \\
=k^{2} p^{2} S_{x} S_{t}\left\{h_{2}(x, t)+\sin \left\{\sum_{i=0}^{\infty}\left[u_{i}(x, t)-v_{i}(x, t)\right]\right\}\right\}
\end{array}\right]\right\} .
\end{array}\right.
$$


Now, by (25), the nonlinear term $\sin (u(x, t)-v(x, t))$ in the systems of equation (38) is decomposed as
Substituting the system of equation (38) into the systems of equation (37), we get

$$
\begin{aligned}
\sin \left(\sum_{i=0}^{\infty}\left(u_{i}(x, t)-v_{i}(x, t)\right)\right)=\sin \left(u_{0}(x, t)-v_{0}(x, t)\right) \\
\quad+\sum_{i=1}^{\infty}\left\{\sin \left(\sum_{r=0}^{i}\left(u_{r}(x, t)-v(x, t)\right)\right)-\sin \left(\left(\sum_{r=0}^{i-1} u_{r}(x, t)-v_{r}(x, t)\right)\right)\right\} .
\end{aligned}
$$

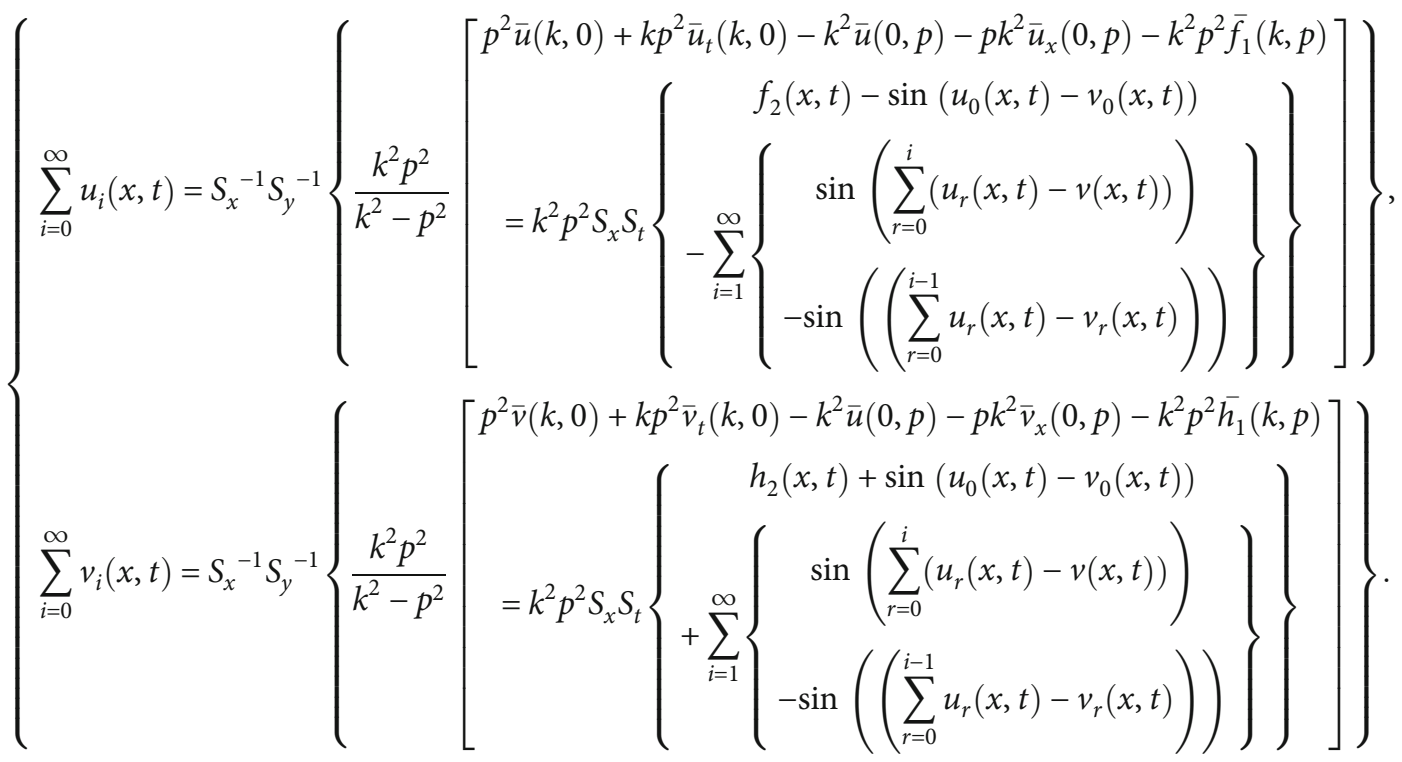

Now, we define the recurrence relation from the systems of equation (40) as follows:

$$
\begin{aligned}
& \left\{\begin{array}{l}
u_{0}(x, t)=S_{x}^{-1} S_{y}^{-1}\left\{\frac{k^{2} p^{2}}{k^{2}-p^{2}}\left[p^{2} \bar{u}(k, 0)+k p^{2} \bar{u}_{t}(k, 0)-k^{2} \bar{u}(0, p)-p k^{2} \bar{u}_{x}(0, p)-k^{2} p^{2} \bar{f}_{1}(k, p)\right]\right\}, \\
v_{0}(x, t)=S_{x}^{-1} S_{y}^{-1}\left\{\frac{k^{2} p^{2}}{k^{2}-p^{2}}\left[p^{2} \bar{v}(k, 0)+k p^{2} \bar{v}_{t}(k, 0)-k^{2} \bar{u}(0, p)-p k^{2} \bar{v}_{x}(0, p)-k^{2} p^{2} \bar{h}_{1}(k, p)\right]\right\},
\end{array}\right. \\
& \left\{\begin{array}{l}
u_{1}(x, t)=S_{x}^{-1} S_{y}^{-1}\left\{\frac{k^{2} p^{2}}{k^{2}-p^{2}}\left[k^{2} p^{2} S_{x} S_{t}\left\{f_{2}(x, t)-\sin \left(u_{0}(x, t)-v_{0}(x, t)\right)\right\}\right]\right\}, \\
v_{1}(x, t)=S_{x}^{-1} S_{y}^{-1}\left\{\frac{k^{2} p^{2}}{k^{2}-p^{2}}\left[k^{2} p^{2} S_{x} S_{t}\left\{h_{2}(x, t)+\sin \left(u_{0}(x, t)-v_{0}(x, t)\right)\right\}\right]\right\},
\end{array}\right.
\end{aligned}
$$

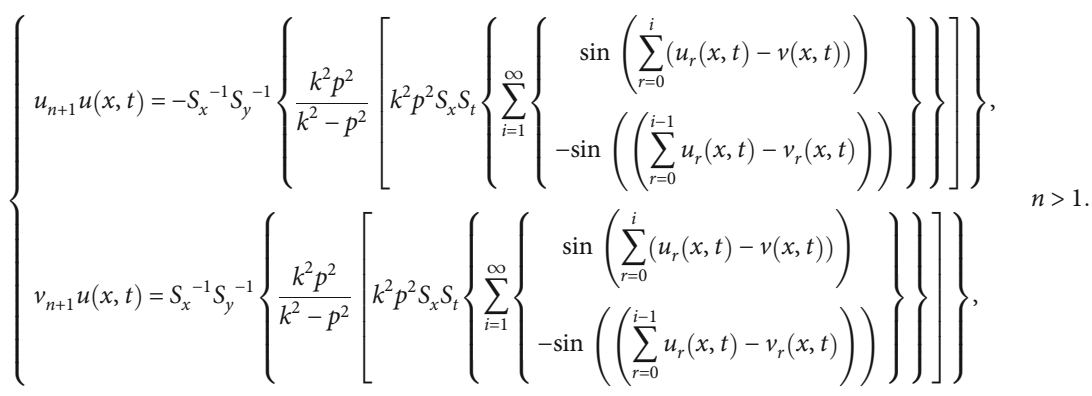


TABLe 1: Double Sumudu transforms of some functions of two variables $[30,41,45,46]$.

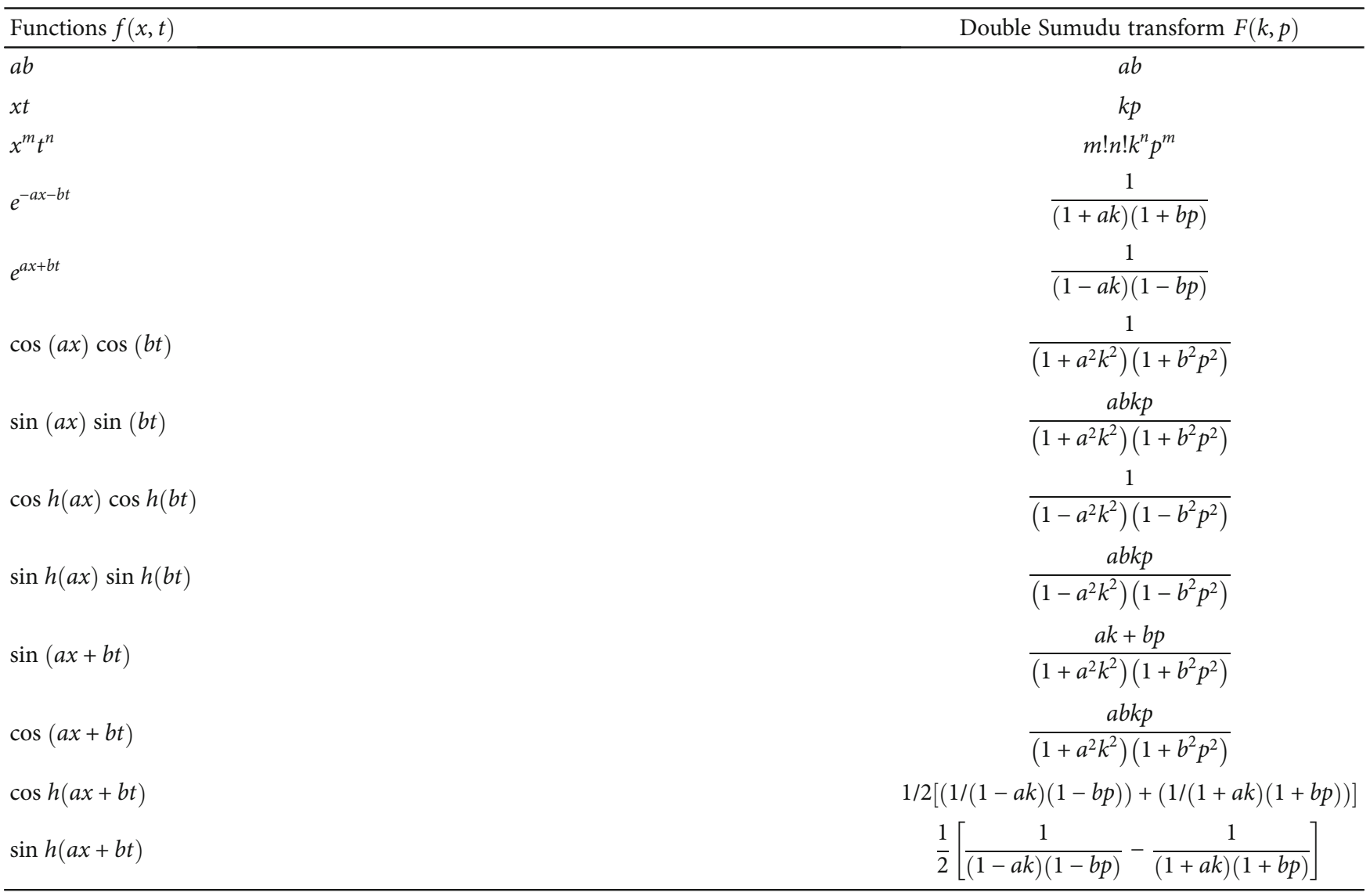

Therefore, the solution of the systems of SGE equations (2)-(4) in series form is given by$$
\left\{\begin{array}{l}
u(x, t)=u_{0}(x, t)+u_{1}(x, t)+u_{2}(x, t)+\cdots+u_{n}(x, t)+\cdots \\
v(x, t)=v_{0}(x, t)+v_{1}(x, t)+v_{2}(x, t)+\cdots+v_{n}(x, t)+\cdots
\end{array} .\right.
$$

Finally, Theorem 13 will be applied to demonstrate the series solution obtained from the new iterative method which is convergent.

\section{Illustrative Examples}

In order to show the validity and effectiveness of the method under consideration, some examples are presented here.

Example 1. Consider the following IBVP for onedimensional coupled sine-Gordon equation on $\Omega=[0, \pi], t$ $>0$ :

$\left\{\begin{array}{l}u_{t t}-u_{x x}=-\sin (u-v)+\sin \left(t \sin x-e^{-t} \sin x\right)+t \sin x, \\ v_{t t}-v_{x x}=\sin (u-v)-\sin \left(t \sin x-e^{-t} \sin x\right)+2 e^{-t} \sin x,\end{array}\right.$ with initial condition

$$
\left\{\begin{array}{l}
u(x, 0)=0, \quad u_{t}(x, 0)=\sin x \\
v(x, 0)=\sin x, \quad v_{t}(x, 0)=-\sin x,
\end{array}\right.
$$

and boundary condition

$$
\left\{\begin{array}{l}
u(0, t)=0, u_{x}(0, t)=t \\
u(0, t)=0, u_{x}(0, t)=e^{-t}
\end{array}\right.
$$

Solution. Applying the properties of double Sumudu transform to both sides of the system of equation (43), we get

$$
\left\{\begin{array}{l}
\frac{1}{k^{2}} \bar{u}(k, p)-\frac{1}{k^{2}} \bar{u}(k, 0)-\frac{1}{k} \bar{u}_{t}(k, 0)-\frac{1}{p^{2}} \bar{u}(k, p)+\frac{1}{p^{2}} \bar{u}(0, p)+\frac{1}{p} \bar{u}_{x}(0, p)+S_{x} S_{t}(\sin (u-v)) \\
=\frac{p k}{1+k^{2}}+S_{x} S_{t}\left(\sin \left(t \sin x-e^{-t} \sin x\right)\right), \\
\frac{1}{k^{2}} \bar{v}(k, p)-\frac{1}{k^{2}} \bar{v}(k, 0)-\frac{1}{k} \bar{v}_{t}(k, 0)-\frac{1}{p^{2}} \bar{v}(k, p)+\frac{1}{p^{2}} \bar{v}(0, p)+\frac{1}{p} \bar{v}_{x}(0, p)-S_{x} S_{t}(\sin (u-v)) \\
=\frac{2}{(1+p)\left(1+k^{2}\right)}-S_{x} S_{t}\left(\sin \left(t \sin x-e^{-t} \sin x\right)\right) .
\end{array}\right.
$$

Multiplying both sides of each equation in the system 
(46) by $k^{2} p^{2}$, we obtain

$$
\left\{\begin{array}{l}
p^{2} \bar{u}(k, p)-p^{2} \bar{u}(k, 0)-k p^{2} \bar{u}_{t}(k, 0)-k^{2} \bar{u}(k, p)+k^{2} \bar{u}(0, p)+p k^{2} \bar{u}_{x}(0, p) \\
=\frac{p^{3} k^{3}}{1+k^{2}}-k^{2} p^{2} S_{x} S_{t}\left(\sin (u-v)-\sin \left(t \sin x-e^{-t} \sin x\right)\right), \\
p^{2} \bar{v}(k, p)-p^{2} \bar{v}(k, 0)-k p^{2} \bar{v}_{t}(k, 0)-k^{2} \bar{v}(k, p)+k^{2} \bar{v}(0, p)+p k^{2} \bar{v}_{x}(0, p) \\
=\frac{2 k^{2} p^{2}}{(1+p)\left(1+k^{2}\right)}+k^{2} p^{2} S_{x} S_{t}\left(\sin (u-v)-\sin \left(t \sin x-e^{-t} \sin x\right)\right) .
\end{array}\right.
$$

Next, applying the single Sumudu transform to the systems of equations (44) and (45), we get

$$
\begin{aligned}
& \left\{\begin{array}{l}
\bar{u}(k, 0)=0, \bar{u}_{t}(k, 0)=\frac{k}{1+k^{2}}, \\
\bar{v}(k, 0)=\frac{k}{1+k^{2}}, \bar{v}_{t}(k, 0)=-\frac{k}{1+k^{2}},
\end{array}\right. \\
& \left\{\begin{array}{l}
\bar{u}(0, p)=0, \bar{u}_{x}(0, p)=p, \\
\bar{v}(0, p)=\frac{k}{1+k^{2}}, \bar{v}_{x}(0, p)=\frac{1}{1+p} .
\end{array}\right.
\end{aligned}
$$

By substituting the system of equations (49) and (48) into the system of equation (47) and simplifying, we obtain

$$
\left\{\begin{array}{l}
\bar{u}(k, p)=\frac{p k}{1+k^{2}}-\frac{k^{2} p^{2}}{k^{2}-p^{2}}\left[S_{x} S_{t}\left\{\sin (u-v)-\sin \left(t \sin x-e^{-t} \sin x\right)\right\}\right], \\
\bar{v}(k, p)=\frac{2 k}{(1+p)\left(1+k^{2}\right)}+\frac{k^{2} p^{2}}{k^{2}-p^{2}}\left[S_{x} S_{t}\left\{\sin (\mathrm{u}-v)-\sin \left(t \sin x-e^{-t} \sin x\right)\right\}\right] .
\end{array}\right.
$$

Applying the inverse double Sumudu transform to (50), we get

$$
\left\{\begin{array}{l}
u(x, t)=t \sin x-S_{x}^{-1} S_{t}^{-1}\left[\frac{k^{2} p^{2}}{k^{2}-p^{2}}\left[S_{x} S_{t}\left\{\sin (u-v)-\sin \left(t \sin x-e^{-t} \sin x\right)\right\}\right]\right] \\
v(x, t)=e^{-t} \sin x+S_{x}^{-1} S_{t}^{-1}\left[\frac{k^{2} p^{2}}{k^{2}-p^{2}}\left[S_{x} S_{t}\left\{\sin (u-v)-\sin \left(t \sin x-e^{-t} \sin x\right)\right\}\right]\right]
\end{array}\right.
$$

Now, applying the new iterative method to a system of equations (51), we obtain the components of the solution as follows:

$$
\begin{aligned}
& \left\{\begin{array}{l}
u_{0}(x, t)=t \sin x, \\
v_{0}(x, t)=e^{-t} \sin x,
\end{array}\right. \\
& \left\{\begin{array}{l}
u_{1}(x, t)=-S_{x}^{-1} S_{t}^{-1}\left[\frac{k^{2} p^{2}}{k^{2}-p^{2}}\left[S_{x} S_{t}\left\{\sin \left(u_{0}(x, t)-v_{0}(x, t)\right)-\sin \left(t \sin x-e^{-t} \sin x\right)\right)\right\}\right], \\
v_{1}(x, t)=S_{x}^{-1} S_{t}^{-1}\left[\frac{k^{2} p^{2}}{k^{2}-p^{2}}\left[S_{x} S_{t}\left\{\sin \left(u_{0}(x, t)-v_{0}(x, t)\right)-\sin \left(t \sin x-e^{-t} \sin x\right)\right\}\right]\right],
\end{array}\right. \\
& \Longrightarrow\left\{\begin{array}{l}
u_{1}(x, t)=-S_{x}^{-1} S_{t}^{-1}\left[\frac{k^{2} p^{2}}{k^{2}-p^{2}}\left[S_{x} S_{t}\left\{\sin \left(t \sin x-e^{-t} \sin x\right)-\sin \left(t \sin x-e^{-t} \sin x\right)\right\}\right]=0\right], \\
v_{1}(x, t)=S_{x}^{-1} S_{t}^{-1}\left[\frac{k^{2} p^{2}}{k^{2}-p^{2}}\left[S_{x} S_{t}\left\{\sin \left(t \sin x-e^{-t} \sin x\right)-\sin \left(t \sin x-e^{-t} \sin x\right)\right\}\right]=0\right],
\end{array}\right. \\
& \left\{u_{n+1} u(x, t)=S_{x}^{-1} S_{y}^{-1}\left\{\frac { k ^ { 2 } p ^ { 2 } } { k ^ { 2 } - p ^ { 2 } } \left[S_{x} S_{t}\left\{\sum_{i=1}^{\infty}\left\{\begin{array}{c}
\sin \left(\sum_{r=0}^{i}\left(u_{r}(x, t)-v_{r}(x, t)\right)\right) \\
-\sin \left(\left(\sum_{r=0}^{i-1} u_{r}(x, t)-v_{r}(x, t)\right)\right)
\end{array}\right\}\right\},\right.\right.\right. \\
& \left\{v_{n+1} u(x, t)=-S_{x}^{-1} S_{y}^{-1}\left\{\frac{k^{2} p^{2}}{k^{2}-p^{2}}\left[S_{x} S_{t}\left\{\sum_{i=1}^{\infty}\left\{\begin{array}{c}
\sin \left(\sum_{r=0}^{i}\left(u_{r}(x, t)-v_{r}(x, t)\right)\right) \\
-\sin \left(\left(\sum_{r=0}^{i-1} u_{r}(x, t)-v_{r}(x, t)\right)\right)
\end{array}\right\}\right\},\right\},\right.\right.
\end{aligned}
$$


For $n=1$,

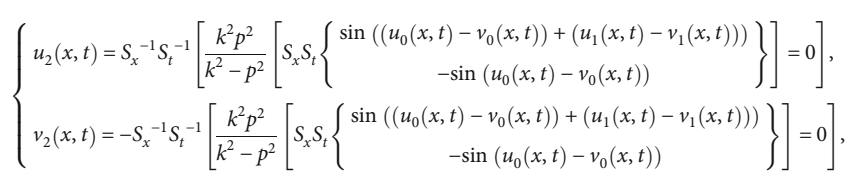

in the same way, we obtain

$$
\left\{\begin{array} { l } 
{ u _ { 3 } ( x , t ) = 0 , } \\
{ v _ { 3 } ( x , t ) = 0 , }
\end{array} \left\{\begin{array}{l}
u_{4}(x, t)=0, \\
v_{4}(x, t)=0,
\end{array}\right.\right.
$$

and so on.

Therefore, the solution of Example 1 in the sense of (42) is

$$
\left\{\begin{array}{l}
u(x, t)=t \sin x, \\
v(x, t)=e^{-t} \sin x,
\end{array}\right.
$$

which is the same as the result obtained by Yildirim et al. [14].

Let us now test the convergence of the obtained series solution. From the system of equation (51), we have $\left\{\begin{array}{l}u_{0}(x, t)=t \sin x, \\ v_{0}(x, t)=e^{-t} \sin x,\end{array}\right.$
$\left\{\begin{array}{l}N(u(x, t))=-S_{x}^{-1} S_{t}^{-1}\left[\frac{k^{2} p^{2}}{k^{2}-p^{2}}\left[S_{x} S_{t}\left\{\sin (u-v)-\sin \left(t \sin x-e^{-t} \sin x\right)\right\}\right]\right], \\ N(v(x, t))=S_{x}^{-1} S_{t}^{-1}\left[\frac{k^{2} p^{2}}{k^{2}-p^{2}}\left[S_{x} S_{t}\left\{\sin (u-v)-\sin \left(t \sin x-e^{-t} \sin x\right)\right\}\right]\right] .\end{array}\right.$

Thus, for all $x, t \geq 0$, we have

$$
\left\{\begin{array}{l}
N\left(u_{0}(x, t)\right)=-S_{x}^{-1} S_{t}^{-1}\left[\frac{k^{2} p^{2}}{k^{2}-p^{2}}\left[S_{x} S_{t}\left\{\sin \left(u_{0}(x, t)-v_{0}(x, t)\right)-\sin \left(t \sin x-e^{-t} \sin x\right)\right\}\right]=0\right], \\
N\left(u_{0}(x, t)\right)=S_{x}^{-1} S_{t}^{-1}\left[\frac{k^{2} p^{2}}{k^{2}-p^{2}}\left[S_{x} S_{t}\left\{\sin \left(u_{0}(x, t)-v_{0}(x, t)\right)-\sin \left(t \sin x-e^{-t} \sin x\right)\right\}\right]=0\right]
\end{array}\right.
$$

Therefore,

$$
\begin{gathered}
\left\{\begin{array}{l}
\left\|N\left(u_{0}(x, t)\right)\right\|=\|0\|=0<\frac{1}{e}, \\
\left\|N\left(v_{0}(x, t)\right)\right\|=\|0\|=0<\frac{1}{e},
\end{array}\right. \\
\left\{\begin{array}{c}
N\left(\frac{\partial}{\partial t} u(x, t)\right)=-S_{x}^{-1} S_{t}^{-1}\left[\frac{k^{2} p^{2}}{k^{2}-p^{2}}\left[S_{x} S_{t}\left\{\begin{array}{c}
\cos (u-v) \frac{\partial}{\partial t}(u-v) \\
-\cos \left(t \sin x-e^{-t} \sin x\right)\left(\sin x+e^{-t} \sin x\right)
\end{array}\right\}\right],\right. \\
N\left(\frac{\partial}{\partial t} v(x, t)\right)=S_{x}^{-1} S_{t}^{-1}\left[\frac{k^{2} p^{2}}{k^{2}-p^{2}}\left[S_{x} S_{t}\left\{\begin{array}{c}
\cos (u-v) \frac{\partial}{\partial t}(u-v) \\
-\cos \left(t \sin x-e^{-t} \sin x\right)\left(\sin x+e^{-t} \sin x\right)
\end{array}\right\}\right]\right]
\end{array}\right.
\end{gathered}
$$


Then,

$$
\begin{aligned}
& \left\{N\left(\frac{\partial}{\partial t} u_{0}(x, t)\right)=-S_{x}^{-1} S_{t}^{-1}\left[\frac{k^{2} p^{2}}{k^{2}-p^{2}}\left[S_{x} S_{t}\left\{\begin{array}{c}
\cos \left(t \sin x-e^{-t} \sin x\right)\left(\sin x+e^{-t} \sin x\right) \\
-c \cos \left(t \sin x-e^{-t} \sin x\right)\left(\sin x+e^{-t} \sin x\right)
\end{array}\right\}\right]=0\right],\right. \\
& \left\{N\left(\frac{\partial}{\partial t} v_{0}(x, t)\right)=S_{x}^{-1} S_{t}^{-1}\left[\frac{k^{2} p^{2}}{k^{2}-p^{2}}\left[S_{x} S_{t}\left\{\begin{array}{c}
\cos \left(t \sin x-e^{-t} \sin x\right)\left(\sin x+e^{-t} \sin x\right) \\
-\cos \left(t \sin x-e^{-t} \sin x\right)\left(\sin x+e^{-t} \sin x\right)
\end{array}\right\}\right]=0\right]\right. \text {, } \\
& \left\{\begin{array}{l}
N\left(\frac{\partial}{\partial x} u(x, t)\right)=-S_{x}^{-1} S_{t}^{-1}\left[\frac{k^{2} p^{2}}{k^{2}-p^{2}}\left[S_{x} S_{t}\left\{\begin{array}{c}
\cos (u-v) \frac{\partial}{\partial x}(u-v) \\
-\cos \left(t \sin x-e^{-t} \sin x\right)\left(t \cos x-e^{-t} \cos x\right)
\end{array}\right\}\right],\right. \\
N\left(\frac{\partial}{\partial x} v(x, t)\right)=S_{x}^{-1} S_{t}^{-1}\left[\frac{k^{2} p^{2}}{k^{2}-p^{2}}\left[S_{x} S_{t}\left\{\begin{array}{c}
\cos (u-v) \frac{\partial}{\partial x}(u-v) \\
-\cos \left(t \sin x-e^{-t} \sin x\right)\left(t \cos x-e^{-t} \cos x\right)
\end{array}\right\}\right]\right]
\end{array}\right.
\end{aligned}
$$

Then,

$$
\left\{\begin{array}{l}
N\left(\frac{\partial}{\partial x} u_{0}(x, t)\right)=-S_{x}^{-1} S_{t}^{-1}\left[\frac{k^{2} p^{2}}{k^{2}-p^{2}}\left[S_{x} S_{t}\left\{\begin{array}{c}
\cos \left(t \sin x-e^{-t} \sin x\right)\left(t \cos x-e^{-t} \cos x\right)- \\
\cos \left(t \sin x-e^{-t} \sin x\right)\left(t \cos x-e^{-t} \cos x\right)
\end{array}\right\}\right]=0\right], \\
N\left(\frac{\partial}{\partial x} v_{0}(x, t)\right)=S_{x}^{-1} S_{t}^{-1}\left[\frac{k^{2} p^{2}}{k^{2}-p^{2}}\left[S_{x} S_{t}\left\{\begin{array}{c}
\cos \left(t \sin x-e^{-t} \sin x\right)\left(t \cos x-e^{-t} \cos x\right)- \\
\cos \left(t \sin x-e^{-t} \sin x\right)\left(t \cos x-e^{-t} \cos x\right)
\end{array}\right\}\right]=0\right] .
\end{array}\right.
$$

Therefore,

$$
\begin{gathered}
\left\{\begin{array}{l}
\left\|N^{\prime}\left(u_{0}(x, t)\right)\right\|=\|0\|=0<\frac{1}{e}, \\
\left\|N^{\prime}\left(v_{0}(x, t)\right)\right\|=\|0\|=0<\frac{1}{e},
\end{array}\right. \\
N\left(\frac{\partial^{2}}{\partial t^{2}} u_{0}(x, t)\right)=-S_{x}{ }^{-1} S_{t}{ }^{-1}\left[\frac{k^{2} p^{2}}{k^{2}-p^{2}}\left[\begin{array}{c}
S_{x} S_{t}\left\{\begin{array}{c}
-\sin \left(t \sin x-e^{-t} \sin x\right)\left(\sin x+e^{-t} \sin x\right)^{2} \\
+\cos \left(t \sin x-e^{-t} \sin x\right)\left(-e^{-t} \sin x\right) \\
\sin \left(t \sin x-e^{-t} \sin x\right)\left(\sin x+e^{-t} \sin x\right)^{2} \\
-\cos \left(t \sin x-e^{-t} \sin x\right)\left(-e^{-t} \sin x\right)
\end{array}\right\}=0 \\
N\left(\frac{\partial^{2}}{\partial t^{2}} v_{0}(x, t)\right)=S_{x}{ }^{-1} S_{t}^{-1}\left[\frac{k^{2} p^{2}}{k^{2}-p^{2}}\left[\begin{array}{c}
-\sin \left(t \sin x-e^{-t} \sin x\right)\left(\sin x+e^{-t} \sin x\right)^{2} \\
+\cos \left(t \sin x-e^{-t} \sin x\right)\left(-e^{-t} \sin x\right) \\
\sin \left(t \sin x-e^{-t} \sin x\right)\left(\sin x+e^{-t} \sin x\right)^{2} \\
-\cos \left(t \sin x-e^{-t} \sin x\right)\left(-e^{-t} \sin x\right)
\end{array}\right\}=0\right.
\end{array}\right],\right.
\end{gathered}
$$




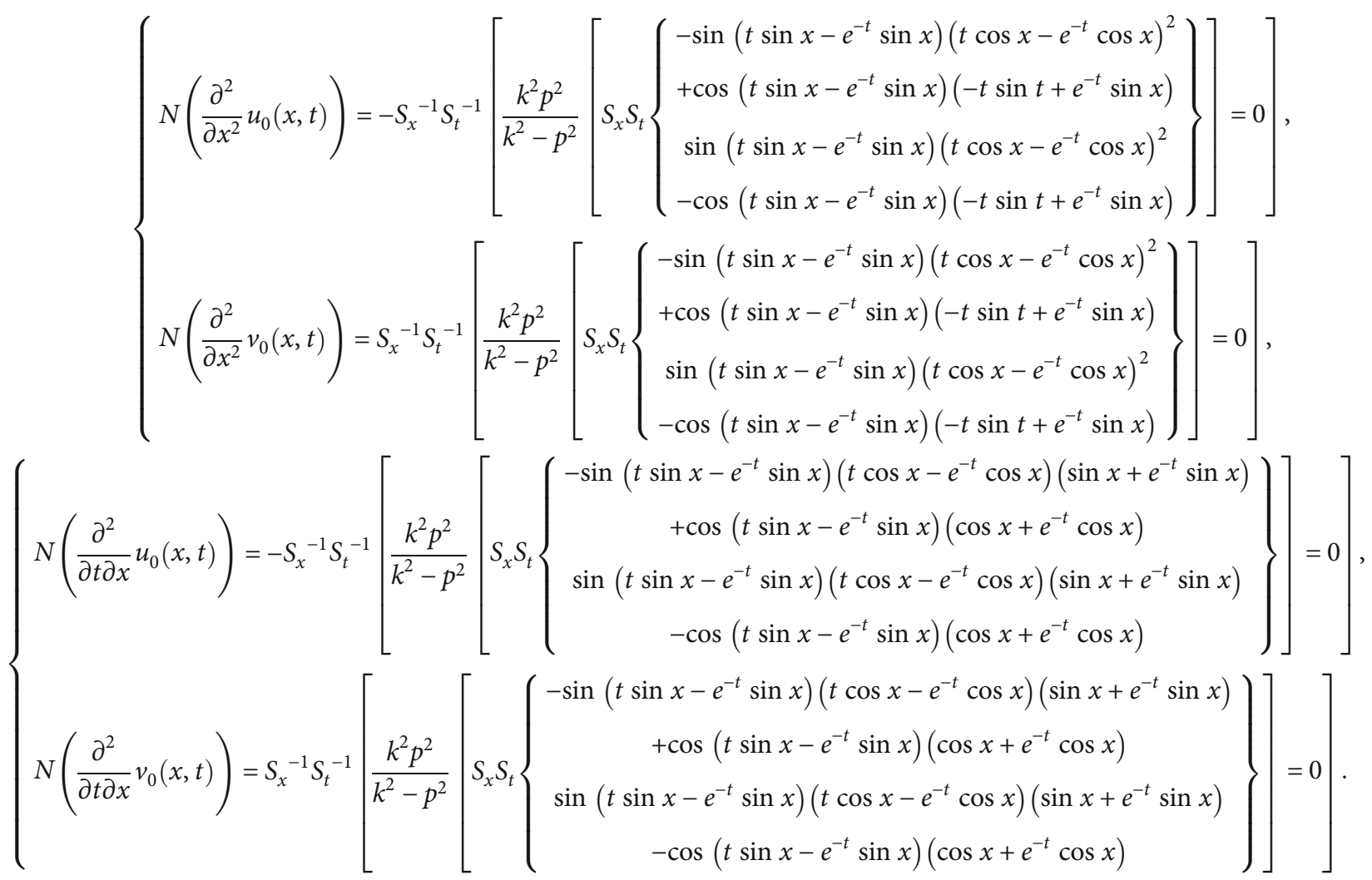

Therefore,

$$
\left\{\begin{array}{l}
\left\|N^{\prime \prime}\left(u_{0}(x, t)\right)\right\|=\|0\|=0<\frac{1}{e} \\
\left\|N^{\prime \prime}\left(v_{0}(x, t)\right)\right\|=\|0\|=0<\frac{1}{e}
\end{array}\right.
$$

Similarly, we have

$$
\left\{\begin{array}{l}
\left\|N^{(3)}\left(u_{0}(x, t)\right)\right\|=\left\|N^{4)}\left(u_{0}(x, t)\right)\right\|=\cdots=\left\|N^{(k)}\left(u_{0}(x, t)\right)\right\|=\|0\|=0<\frac{1}{e}, \\
\left\|N^{(3)}\left(v_{0}(x, t)\right)\right\|=\left\|N^{4)} v_{0}(x, t)\right\|=\cdots=\left\|N^{(k)}\left(v_{0}(x, t)\right)\right\|=\|0\|=0<\frac{1}{e},
\end{array}\right.
$$

for all $k \geq 0$ by the principle of mathematical induction.

As the conditions of Theorem 13 are satisfied, the series solution obtained by the new iterative method is convergent on the domain of interest.

Example 2. Consider the following IBVP for onedimensional coupled sine-Gordon equation on $\Omega=[0, \pi], t$ $>0$ :

$$
\left\{\begin{array}{l}
u_{t t}-u_{x x}=-\sin (u-v)+\sin (\cos (x+2 t)-\sin x \sinh (t))-3 \cos (x+2 t), \\
v_{t t}-v_{x x}=\sin (u-v)-\sin (\cos (x+2 t)-\sin x \sinh (t))+2 \sin x \sinh (t),
\end{array}\right.
$$

with initial condition

$$
\left\{\begin{array}{l}
u(x, 0)=\cos x, \quad u_{t}(x, 0)=-2 \sin x \\
v(x, 0)=0, \quad v_{t}(x, 0)=\sin x
\end{array}\right.
$$

and boundary condition

$$
\left\{\begin{array}{l}
u(0, t)=\cos (2 t), u_{x}(0, t)=-\sin (2 t) \\
u(0, t)=0, u_{x}(0, t)=\sin h(t)
\end{array}\right.
$$

Solution. Applying the properties of the double Sumudu transform to both sides of the system of equations (66), we get 


$$
\left\{\begin{array}{l}
\frac{1}{k^{2}} \bar{u}(k, p)-\frac{1}{k^{2}} \bar{u}(k, 0)-\frac{1}{k} \bar{u}_{t}(k, 0)-\frac{1}{p^{2}} \bar{u}(k, p)+\frac{1}{p^{2}} \bar{u}(0, p)+\frac{1}{p} \bar{u}_{x}(0, p)+S_{x} S_{t}(\sin (u-v))=-\frac{6 k p}{\left(1+k^{2}\right)\left(1+4 p^{2}\right)}+S_{x} S_{t}(\sin (\cos (x+2 t)-\sin x \sinh (t))) \\
\frac{1}{k^{2}} \bar{v}(k, p)-\frac{1}{k^{2}} \bar{v}(k, 0)-\frac{1}{k} \bar{v}_{t}(k, 0)-\frac{1}{p^{2}} \bar{v}(k, p)+\frac{1}{p^{2}} \bar{v}(0, p)+\frac{1}{p} \bar{v}_{x}(0, p)-S_{x} S_{t}(\sin (u-v))=\frac{2 k p}{\left(1+k^{2}\right)\left(1-p^{2}\right)}+S_{x} S_{t}(\sin (\cos (x+2 t)-\sin x \sinh (t))) .
\end{array}\right.
$$

Multiplying both sides of each equation in the system of equation (69) by $k^{2} p^{2}$, we get

$$
\left\{\begin{array}{l}
p^{2} \bar{u}(k, p)-p^{2} \bar{u}(k, 0)-k p^{2} \bar{u}_{t}(k, 0)-k^{2} \bar{u}(k, p)+k^{2} \bar{u}(0, p)+p k^{2} \bar{u}_{x}(0, p) \\
=-\frac{6 p^{3} k^{3}}{\left(1+k^{2}\right)\left(1+4 p^{2}\right)}-k^{2} p^{2} S_{x} S_{t}(\sin (u-v)-\sin (\cos (x+2 t)-\sin x \sinh (t))), \\
p^{2} \bar{v}(k, p)-p^{2} \bar{v}(k, 0)-k p^{2} \bar{v}_{t}(k, 0)-k^{2} \bar{v}(k, p)+k^{2} \bar{v}(0, p)+p k^{2} \bar{v}_{x}(0, p) \\
=\frac{2 p^{3} k^{3}}{\left(1+k^{2}\right)\left(1-p^{2}\right)}+k^{2} p^{2} S_{x} S_{t}(\sin (u-v)-\sin (\cos (x+2 t)-\sin x \sinh (t))) .
\end{array}\right.
$$

Next, applying a single Sumudu transform to the systems of equations (67) and (68), we obtain

$$
\begin{gathered}
\left\{\begin{array}{l}
\bar{u}(k, 0)=\frac{1}{1+k^{2}}, \bar{u}_{t}(k, 0)=\frac{-2 k}{1+k^{2}}, \\
\bar{v}(k, 0)=0, \bar{v}_{t}(k, 0)=\frac{k}{1+k^{2}},
\end{array}\right. \\
\left\{\begin{array}{l}
\bar{u}(0, p)=\frac{1}{1+4 p^{2}}, \bar{u}_{x}(0, p)=\frac{-2 p}{1+4 p^{2}}, \\
\bar{v}(0, p)=\frac{k}{1+k^{2}}, \bar{v}_{x}(0, p)=\frac{p}{1-4 p^{2}} .
\end{array}\right.
\end{gathered}
$$

By substituting the systems of equations (72) and (71) into the system of equation (70) and simplifying, we obtain

$$
\left\{\begin{array}{l}
\bar{u}(k, p)=\frac{2 k p}{\left(1+k^{2}\right)\left(1+4 p^{2}\right)}-\frac{k^{2} p^{2}}{k^{2}-p^{2}}\left[S_{x} S_{t}\{\sin (u-v)-\sin (\cos (x+2 t)-\sin x \sinh (t))\}\right] \\
\bar{v}(k, p)=\frac{k p}{\left(1+k^{2}\right)\left(1-p^{2}\right)}+\frac{k^{2} p^{2}}{k^{2}-p^{2}}\left[S_{x} S_{t}\{\sin (u-v)-\sin (\cos (x+2 t)-\sin x \sinh (t))\}\right]
\end{array}\right.
$$

Applying inverse double Sumudu transform to (73), we obtain

$$
\left\{\begin{array}{l}
u(x, t)=\cos (x+2 t)-S_{x}^{-1} S_{t}^{-1}\left[\frac{k^{2} p^{2}}{k^{2}-p^{2}}\left[S_{x} S_{t}\{\sin (u-v)-\sin (\cos (x+2 t)-\sin x \sinh (t))\}\right]\right] \\
v(x, t)=\sin x \sinh (t)+S_{x}^{-1} S_{t}^{-1}\left[\frac{k^{2} p^{2}}{k^{2}-p^{2}}\left[S_{x} S_{t}\{\sin (u-v)-\sin (\cos (x+2 t)-\sin x \sinh (t))\}\right]\right]
\end{array}\right.
$$

Now, applying the new iterative method to the system of equations (74), we obtain the components of the solution as follows:

$$
\begin{aligned}
& \left\{\begin{array}{l}
u_{0}(x, t)=\cos (x+2 t), \\
v_{0}(x, t)=\sin x \sinh (t),
\end{array}\right. \\
& \left\{\begin{array}{l}
\left.u_{1}(x, t)=-S_{x}^{-1} S_{t}^{-1}\left[\frac{k^{2} p^{2}}{k^{2}-p^{2}}\left[S_{x} S_{t}\left\{\sin \left(u_{0}(x, t)-v_{0}(x, t)\right)-\sin (\cos (x+2 t)-\sin x \sinh (t))\right)\right\}\right]\right], \\
v_{1}(x, t)=S_{x}^{-1} S_{t}^{-1}\left[\frac{k^{2} p^{2}}{k^{2}-p^{2}}\left[S_{x} S_{t}\left\{\sin \left(u_{0}(x, t)-v_{0}(x, t)\right)-\sin (\cos (x+2 t)-\sin x \sinh (t))\right\}\right]\right],
\end{array}\right. \\
& \Longrightarrow\left\{\begin{array}{l}
u_{1}(x, t)=-S_{x}^{-1} S_{t}^{-1}\left[\frac{k^{2} p^{2}}{k^{2}-p^{2}}\left[S_{x} S_{t}\left\{\sin \left(t \sin x-e^{-t} \sin x\right)-\sin \left(t \sin x-e^{-t} \sin x\right)\right\}\right]=0\right], \\
v_{1}(x, t)=S_{x}^{-1} S_{t}^{-1}\left[\frac{k^{2} p^{2}}{k^{2}-p^{2}}\left[S_{x} S_{t}\left\{\sin \left(t \sin x-e^{-t} \sin x\right)-\sin \left(t \sin x-e^{-t} \sin x\right)\right\}\right]=0\right],
\end{array}\right.
\end{aligned}
$$

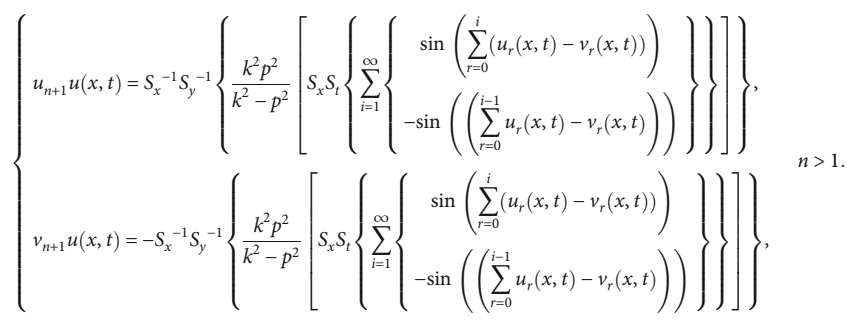

For $n=1$,

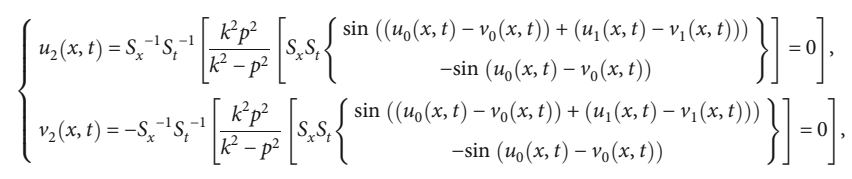

in the same way, we obtain

$$
\left\{\begin{array} { l } 
{ u _ { 3 } ( x , t ) = 0 , } \\
{ v _ { 3 } ( x , t ) = 0 , }
\end{array} \left\{\begin{array}{l}
u_{4}(x, t)=0 \\
v_{4}(x, t)=0
\end{array}\right.\right.
$$

and so on.

Therefore, the solution of Example 2 in the sense of (40) is

$$
\left\{\begin{array}{l}
u(x, t)=\cos (x+2 t), \\
v(x, t)=\sin x \sinh (t) .
\end{array}\right.
$$

Let us now test the convergence of the obtained series 
solution. From the system of equation (74), we have

$$
\begin{gathered}
\left\{\begin{array}{l}
u_{0}(x, t)=\cos (x+2 t), \\
v_{0}(x, t)=\sin x \sinh (t),
\end{array}\right. \\
\left\{\begin{array}{l}
N(u(x, t))=-S_{x}^{-1} S_{t}^{-1}\left[\frac{k^{2} p^{2}}{k^{2}-p^{2}}\left[S_{x} S_{t}\{\sin (u-v)-\sin (\cos (x+2 t)-\sin x \sinh (t))\}\right]\right] \\
N(v(x, t))=S_{x}^{-1} S_{t}^{-1}\left[\frac{k^{2} p^{2}}{k^{2}-p^{2}}\left[S_{x} S_{t}\{\sin (u-v)-\sin (\cos (x+2 t)-\sin x \sinh (t))\}\right]\right] .
\end{array}\right.
\end{gathered}
$$

Thus, for all $x, t \geq 0$, we have

$$
\left\{\begin{array}{l}
N\left(u_{0}(x, t)\right)=-S_{x}^{-1} S_{t}^{-1}\left[\frac{k^{2} p^{2}}{k^{2}-p^{2}}\left[S_{x} S_{t}\left\{S_{x} S_{t}\left\{\begin{array}{c}
\sin (\cos (x+2 t)-\sin x \sinh (t)) \\
-\sin (\cos (x+2 t)-\sin x \sinh (t))
\end{array}\right\}\right\}\right]=0\right] \\
N\left(u_{0}(x, t)\right)=S_{x}^{-1} S_{t}^{-1}\left[\frac{k^{2} \mathrm{p}^{2}}{k^{2}-p^{2}}\left[S_{x} S_{t}\left\{\begin{array}{c}
\sin (\cos (x+2 t)-\sin x \sinh (t)) \\
-\sin (\cos (x+2 t)-\sin x \sinh (t))
\end{array}\right\}\right]=0\right] .
\end{array}\right.
$$

Therefore,

$$
\begin{gathered}
\left\{\begin{array}{l}
\left\|N\left(u_{0}(x, t)\right)\right\|=\|0\|=0<\frac{1}{e}, \\
\left\|N\left(v_{0}(x, t)\right)\right\|=\|0\|=0<\frac{1}{e},
\end{array}\right. \\
\left\{\begin{array}{l}
N\left(\frac{\partial}{\partial t} u(x, t)\right)=-S_{x}^{-1} S_{t}^{-1}\left[\frac{k^{2} p^{2}}{k^{2}-p^{2}}\left[S_{x} S_{t}\left\{\begin{array}{c}
\cos (u-v) \frac{\partial}{\partial t}(u-v)+ \\
\cos (\cos (x+2 t)-\sin x \sinh (t)) \\
(-2 \cos (x+2 t)-\sin x i n h(t))
\end{array}\right\}\right)\right] \\
N\left(\frac{\partial}{\partial t} v(x, t)\right)=S_{x}^{-1} S_{t}^{-1}\left[\frac{k^{2} p^{2}}{k^{2}-p^{2}}\left[S_{x} S_{t}\left\{\begin{array}{c}
\cos (u-v) \frac{\partial}{\partial t}(u-v)+ \\
\cos (\cos (x+2 t)-\sin x \sinh (t)) \\
(-2 \cos (x+2 t)-\sin x i n h(t))
\end{array}\right)\right]\right]
\end{array}\right.
\end{gathered}
$$

Then,

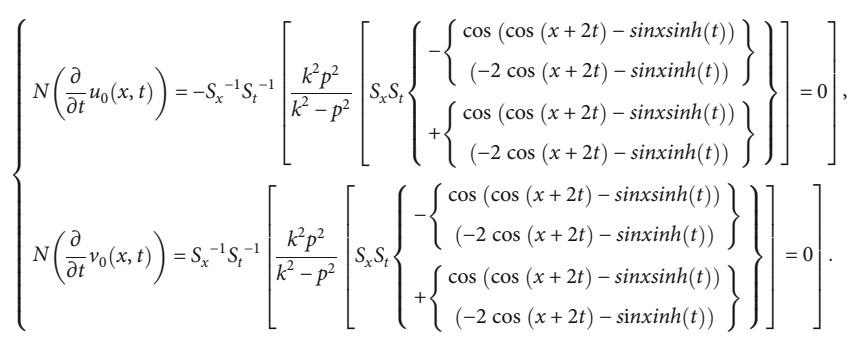

Therefore,

$$
\left\{\begin{array}{l}
\left\|N^{\prime}\left(u_{0}(x, t)\right)\right\|=\|0\|=0<\frac{1}{e}, \\
\left\|N^{\prime}\left(v_{0}(x, t)\right)\right\|=\|0\|=0<\frac{1}{e} .
\end{array}\right.
$$

Similarly, we have

$$
\left\{\begin{array}{l}
\left\|N^{\prime \prime}\left(u_{0}(x, t)\right)\right\|=\left\|N^{(3)}\left(u_{0}(x, t)\right)\right\|=\left\|N^{4)}\left(u_{0}(x, t)\right)\right\|=\cdots=\left\|N^{(k)}\left(u_{0}(x, t)\right)\right\|=\|0\|=0<\frac{1}{e}, \\
\left\|N^{\prime \prime}\left(v_{0}(x, t)\right)\right\|=\left\|N^{(3)}\left(v_{0}(x, t)\right)\right\|=\left\|N^{4)} v_{0}(x, t)\right\|=\cdots=\left\|N^{(k)}\left(v_{0}(x, t)\right)\right\|=\|0\|=0<\frac{1}{e},
\end{array}\right.
$$

for all $k \geq 0$ by the principle of mathematical induction.

As the conditions of Theorem 13 are satisfied, the series solution obtained by the new iterative method is convergent on the domain of interest.

\section{Conclusion}

The double Sumudu transform method has been known to be a powerful tool for solving many fractional partial differential equations, integral equations, and so many other equations. In this paper, we have presented a new method, that is, the amalgamation of double Sumudu transform and a numerical method which is known as an iterative method to obtain the exact solution of one dimensional coupled nonlinear sine-Gordon equation. From the solutions of illustrative Examples 1 and 2, we can see that the decomposition

$$
\left\{\begin{array}{l}
u(x, t)=\sum_{i=0}^{\infty} u_{i}(x, t)=u_{0}(x, t)+u_{1}(x, t)+u_{2}(x, t)+\cdots+u_{n}(x, t)+\cdots, \\
v(x, t)=\sum_{i=0}^{\infty} v_{i}(x, t)=v_{0}(x, t)+v_{1}(x, t)+v_{2}(x, t)+\cdots+v_{n}(x, t)+\cdots,
\end{array}\right.
$$

consists only of one term, i.e.,

$$
\left\{\begin{array}{l}
u(x, t)=u_{0}(x, t) \\
v(x, t)=s v_{0}(x, t)
\end{array}\right.
$$

From this study, we concluded that double Sumudu transform linked with an iterative method finds quite practical analytical results with less computational work.

\section{Data Availability}

No data were used to support the study.

\section{Conflicts of Interest}

The author declares that there is no conflict of interest regarding the preparation of this research paper.

\section{Acknowledgments}

The author would like to thank Mizan Tepi University, College of Natural Sciences, and the Department of Mathematics for providing the necessary resources during conducting this research. 


\section{References}

[1] K. R. Khusnutdinova and D. E. Pelinovsky, "On the exchange of energy in coupled Klein-Gordon equations," Wave Motion, vol. 38, no. 1, pp. 1-10, 2003.

[2] T. A. Kontorova and Y. I. Frenkel, "On the theory of plastic deformation and twinning I, II," Zhurnal Eksperimental'noi $i$ Teoreticheskoi Fiziki, vol. 8, pp. 89-95, 1938.

[3] O. M. Braun and Y. S. Kivshar, "Nonlinear dynamics of the Frenkel-Kontorova model," Physics Reports, vol. 306, no. 1-2, pp. 1-108, 1998.

[4] S. Yomosa, "Soliton excitations in deoxyribonucleic acid (DNA) double helices," Physical Review A, vol. 27, no. 4, pp. 2120-2125, 1983.

[5] J. K. Perring and T. H. R. Skyrme, "A model unified field equation,” Nuclear Physics, vol. 31, pp. 550-555, 1962.

[6] G. B. Whitham, "Linear and nonlinear waves, reprint of the 1974 original," in Pure and Applied Mathematics (New York), A Wiley-Interscience Publication, John Wiley \& Sons, Inc., New York, 1999.

[7] D. Li, H. Lai, and C. Lin, "Mesoscopic simulation of the two-component system of coupled sine-Gordon equations with lattice Boltzmann method," Entropy, vol. 21, no. 6, p. 542, 2019.

[8] A. H. Salas, "Exact solutions of coupled sine-Gordon equations," Nonlinear Analysis: Real World Applications, vol. 11, no. 5, pp. 3930-3935, 2010.

[9] Y.-M. Zhao, "Exact solutions of coupled sine-gordon equations using the simplest equation method," Journal of Applied Mathematics, vol. 2014, Article ID 534346, 5 pages, 2014.

[10] Y.-M. Zhao, H. Liu, and Y.-J. Yang, "Applications of the Jacobi elliptic function expansion method for obtaining traveling wave solutions of coupled sine-Gordon equations," Mathematical Sciences Research Journal, vol. 15, no. 3, pp. 80-90, 2011.

[11] Y.-M. Zhao, H. Liu, and Y.-J. Yang, "Exact solutions for the coupled sine-Gordon equations by a new hyperbolic auxiliary function method," Applied Mathematical Sciences, vol. 5, no. 33-36, pp. 1621-1629, 2011.

[12] Y.-M. Zhao, W. Li, and Y.-J. Yang, "New exact solutions of coupled sine-Gordon equations using symbolic computation," Mathematical Sciences Research Journal, vol. 14, no. 4, pp. 79$86,2010$.

[13] A. Sadighi, D. D. Ganji, and B. Ganjavi, "Traveling wave solutions of the sine-Gordon and the coupled sine-Gordon equations using the homotopy-perturbation method," Transaction B: Mechanical Engineering, vol. 16, no. 2, pp. 189-195, 2009.

[14] O. Yildirim, M. Uzun, and O. Altun, "On the numerical solution of nonlinear system of coupled sine-Gordon equations," AIP Conference Proceedings, vol. 1997, article 020062, 2018.

[15] K. Hosseini, P. Mayeli, and D. Kumar, "New exact solutions of the coupled sine-Gordon equations in nonlinear optics using the modified Kudryashov method," Journal of Modern Optics, vol. 65, no. 3, pp. 361-364, 2018.

[16] L. Liu and C. Li, "Coupled sine-Gordon systems in DNA dynamics," Advances in Mathematical Physics, vol. 2018, Article ID 4676281, 7 pages, 2018.

[17] S. Latifi and M. Delkhosh, "Generalized Lagrange JacobiGauss-Lobatto vs Jacobi-Gauss-Lobatto collocation approximations for solving $(2+1)$-dimensional sine-Gordon equa- tions," Mathematical Methods in the Applied Sciences, vol. 43, no. 4, pp. 2001-2019, 2020.

[18] Y. Zhao, "New exact solutions for a higher-order wave equation of $\mathrm{KdV}$ type using the multiple simplest equation method," Journal of Applied Mathematics, vol. 2014, Article ID 848069, 13 pages, 2014.

[19] G. K. Watugala, "Sumudu transform: a new integral transform to solve differential equations and control engineering problems," International Journal of Mathematical Education in Science and Technology, vol. 24, no. 1, pp. 35-43, 1993.

[20] A. Al-Nemrat and Z. Zainuddin, "Homotopy perturbation Sumudu transform method for solving nonlinear boundary value problems," AIP Conference Proceedings, vol. 1974, article 020109, 2018.

[21] A. D. Chindhe, "Applications of generalized Sumudu transform," Innovare Journal of Sciences, vol. 8, no. 7, pp. 116$122,2020$.

[22] F. B. M. Belgacem and R. Silambarasan, "Sumudu transform of Dumont bimodular Jacobi elliptic functions for arbitrary powers," AIP Conference Proceedings, vol. 2017, no. 1, 13 pages, 2017.

[23] G. K. Watugala, "The Sumudu transform for functions of two variables," Mathematical Engineering in Industry, vol. 18, no. 4, pp. 293-302, 2002.

[24] Z. Ahmeda, M. I. Idreesb, F. B.-M. Belgacemc, and Z. Perveen, "On the convergence of double Sumudu transform," Journals of Nonlinear Sciences and Applications, vol. 13, pp. 154-162, 2019.

[25] H. S. Kadhem and S. Q. Hasan, "Numerical double Sumudu transform for nonlinear mixed fractional partial differential equations," Journal of Physics: Conference Series, vol. 1279, article 012048, 2019.

[26] S. B. Kiwne and S. M. Sonawane, "Applications of sumudu transform of two variable functions with Hermite polynomial to partial differential equations," Advances in Mathematics: Scientific Journal, vol. 9, no. 1, pp. 107-118, 2020.

[27] A. M. Wazwaz, Partial Differential Equations and Solitary Wave's Theory, Springer, New York/Dordrecht Heidelberg, 2009.

[28] S. Vilu, R. R. Ahmad, and U. K. S. Din, "Variational iteration method and Sumudu transform for solving delay differential equation," International Journal of Differential Equations, vol. 2019, Article ID 6306120, 6 pages, 2019.

[29] T. R. Ramesh Rao, "Exact solution of gas dynamics equations through reduced differential transform and Sumudu transform linked with Pad》 approximants," Journal of Physics: Conference Series, vol. 1000, no. 1, article 012107, 2018.

[30] T. M. Elzaki, E. M. Hilal, and J. S. Arabia, "Solution of telegraph equation by modified of double Sumudu transform (Elzaki Transform)," Mathematical Theory and Modeling, vol. 2, no. 4, pp. 95-103, 2012.

[31] D. Baleanu and H. K. Jassim, "Exact solution of twodimensional fractional partial differential equations," Fractal and Fractional, vol. 4, no. 2, p. 21, 2020.

[32] E. A. Yousif and S. H. M. Hamed, "Solution of nonlinear fractional differential equations using the Homotopy perturbation Sumudu transform method," Applied Mathematical Sciences, vol. 8, no. 44, pp. 2195-2210, 2014.

[33] V. Daftardar-Gejji and H. Jafari, "An iterative method for solving nonlinear functional equations," Journal of Mathematical Analysis and Applications, vol. 316, no. 2, pp. 753-763, 2006. 
[34] L. Ali, S. Islam, T. Gul, and I. S. Amiri, "Solution of nonlinear problems by a new analytical technique using Daftardar-Gejji and Jafari polynomials," Advances in Mechanical Engineering, vol. 11, no. 12, 10 pages, 2019.

[35] M. A. AL-Jawary, G. H. Radhi, and J. Ravnik, "Daftardar-Jafari method for solving nonlinear thin film flow problem," Arab Journal of Basic and Applied Sciences, vol. 25, no. 1, pp. 20 27, 2018

[36] M. A. AL-Jawary, "Analytic solutions for solving fourth-order parabolic partial differential equations with variable coefficients," International Journal of Advanced Scientific and Technical Research, vol. 3, no. 5, pp. 531-535, 2015.

[37] M. S. Al-luhaibi, "New iterative method for fractional gas dynamics and coupled Burger's equations," The Scientific World Journal, vol. 2015, Article ID 153124, 8 pages, 2015.

[38] R. R. Dhunde and G. L. Waghamare, "Analytical solution of the non-linear Klein-Gordon equation using double Laplace transform and iterative method," American Journal of Computational and Applied Mathematics, vol. 6, no. 6, p. 195, 2016.

[39] R. R. Dhunde and G. L. Waghamare, "Double Laplace transform combined with iterative method for solving non-linear telegraph equation," Journal of the Indian Mathematical Society, vol. 83, no. 3-4, pp. 221-230, 2016.

[40] M. O. Eshag, "On double Laplace transform and double Sumudu transform," American Journal of Engineering Research (AJER), vol. 6, no. 5, pp. 312-317, 2017.

[41] A. K. Man, H. Eltayeb, and K. A. M. Atan, "A note on the comparison between Laplace and Sumudu transforms," Bulletin of the Iranian Mathematical Society, vol. 37, no. 1, pp. 131-141, 2011.

[42] S. A. Ahmed, T. M. Elzaki, M. Elbadri, and M. Z. Mohamed, "Solution of partial differential equations by new double integral transform (Laplace - Sumudu transform)," Ain Shams Engineering Journal, vol. 12, no. 4, pp. 4045-4049, 2021.

[43] S. A. Ahmed, T. M. Elzaki, and A. A. Hassan, "Solution of integral differential equations by new double integral transform (Laplace-Sumudu transform)," Abstract and Applied Analysis, vol. 2020, Article ID 4725150, 7 pages, 2020.

[44] J. M. Tchuenche and N. S. Mbare, "An application of the double sumudu transform," Applied Mathematical Sciences, vol. 1, no. 1, pp. 31-39, 2007.

[45] H. Eltayeb and A. Kiliçman, "On double Sumudu transform and double Laplace transform," Malaysian Journal of Mathematical Sciences, vol. 4, no. 1, pp. 17-30, 2010.

[46] M. S. Mechee and A. J. Naeemah, "A study of double Sumudu transform for solving differential equations with some applications," International Journal of Engineering and Information Systems (IJEAIS), vol. 4, no. 1, pp. 20-27, 2020. 